\title{
A Morphological Study of the Effect of Carbon Nanotube Filler on Tribology of Phenol/Formaldehyde Resin-based Composites
}

\author{
Akira IGARAShI, ${ }^{2}$ Toshinari Terasawa,,${ }^{1}$ Mihoko KANIE, ${ }^{1}$ \\ Takeshi YAMANOBE, ${ }^{1}$ and Tadashi KOMOTO ${ }^{1, \dagger}$ \\ ${ }^{1}$ Department of Chemistry, Gunma University, 1-5-1 Tenjin-cho, Kiryu 376-8515, Japan \\ ${ }^{2}$ Tomo Industrial Technology Center, 1058-5 Yoshizawa-cho, Ota 373-0019, Japan
}

(Received January 25, 2005; Accepted April 11, 2005; Published July 15, 2005)

\begin{abstract}
A morphological study was made on preparation of phenol/formaldehyde resin-based composites with carbon nanotube and their tribological properties. It was found that a dispersion of the carbon nanotubes in the matrix resin is obtained by mixing them in a highly viscous molten or concentrated state of the phenolic resin as was revealed by transmission electron microscopy. The friction and wear mechanisms of the neat phenolic resin and the nanocomposites are proposed based on the results obtained by morphological and mechanical measurements. [DOI 10.1295/polymj.37.522]

KEY WORDS Phenol Resin / Nanocomposite / Carbon Nanotube / Friction / Wear / TEM /
\end{abstract}

One of the advantages of thermoplastic polymers, polymer alloys and their composites as tribomaterials is the self-lubricating properties without lubricants. For example, ultrahigh molecular weight polyethylene (UHMWPE) has so low friction coefficient and specific wear rate as to be widely used as lining materials coated on metals, an artificial joint and others. Polyacetals are also used as plastic gears of automobiles, printers and others with and without lubricants. Other various polymers are also applied to industrial uses as polymer alloys and composites.

On the other hand, thermosetting resins such as phenol/formaldehyde (phenolic) resin are used as an adhesive component in brake materials ${ }^{1}$ in which are contained metallic, inorganic and polymeric fibers and various metallic and inorganic powders. Thus, phenolic resin is used as an organic adhesive in organic and inorganic composite materials. Interest has been recently focused on tribomaterials based on phenolic resin composites. For example, Haraguchi et $a l .^{2-4}$ developed a hybrid tribomaterial consisting of phenolic resin and silica, the latter being prepared from alkoxy silicate in the resin matrix. It was revealed that friction coefficient of the hybrid material decreased with increasing silica content up to $c a$. $3 \mathrm{wt} \%$. The size of silica particles can be controlled in nanometer scale, indicating nanocomposites.

Polymer-based nanocomposites as well as metalbased ones have been increasingly expected not only for tribomaterials but also for other industrial materials. For example, nylon-based nanocomposites have been recently developed, where clays and other in- organic materials are mainly used as fillers.

Carbon nanotubes are also promising fillers for polymer-based nanocomposites. There have been reported many papers on polymer-based nanocomposites with carbon nanotube fillers exclusively for these few years. Most of them are relevant to preparations, structures and mechanical, electronic and other properties of the nanocomposites as reviewed. ${ }^{5,6}$

Of these nanocomposites with carbon nanotubes, tribological behaviors were studied on some thermoplastic polymer-based nanocomposites. ${ }^{7,8}$ Very few studies on thermosetting polymers such as phenolic resin-based nanocomposites with carbon nanotubes have been reported so far. We are interested in tribology of the phenolic resin nanocomposites with reference to structure and some mechanical properties and morphology.

In this paper, we will report on a preliminary study on preparation of the phenolic resin-based nanocomposites with carbon nanotubes and their morphology and tribological behaviors.

\section{EXPERIMENTAL}

\section{Preparation of Phenolic Resin Oligomer}

As phenolic resin oligomers prepared from phenol and formaldehyde melt below $c a .80^{\circ} \mathrm{C}$, we attempted first to prepare the oligomer and then to obtain a homogeneous mixture with carbon nanotube in the molten state above $100^{\circ} \mathrm{C}$, giving rise to cross-linked phenolic resin by a further reaction in which carbon nanotubes are dispersed.

${ }^{\dagger}$ To whom correspondence should be addressed (Tel: +81-277-30-1330, E-mail: komoto@cc.gunma-u.ac.jp). 
$188 \mathrm{~g}$ of phenol and $37 \mathrm{wt} \%$ aqueous formaldehyde were mixed in a round separable flask equipped with a mechanical stirrer, a dropping funnel and a thermometer in a water bath. $9.8 \mathrm{~g}$ of $25 \%$ ammonia water was added in dropwise into the mixture at a rate of $2 \mathrm{~mL} /$ min with stirring at ambient temperature. Then the mixture was heated at a rate of $1-2^{\circ} \mathrm{C} / \mathrm{min}$ up to $95^{\circ} \mathrm{C}$ and kept at the temperature with stirring so that the condensation reaction proceeded. After the mixture turned turbid in $10-15 \mathrm{~min}$ at the temperature, condensed water was removed by distillation under a reduced pressure until the mixture became transparent to a large extent, allowing spontaneous decrease in temperature of the mixed solution down to $c a .40^{\circ} \mathrm{C}$. Into the reaction product thus obtained was added a given amount of methanol $10 \mathrm{wt} \%$-equivalent to the product to keep the liquid state of phenolic resin oligomer even at room temperature and below. The oligomer/methanol mixture was kept in a flask at ca. $4{ }^{\circ} \mathrm{C}$ to avoid further reaction.

\section{Further Reaction of Phenolic Resin Oligomer and Preparation of Sheet Specimen}

Phenolic resin oligomer undergoes a further reaction by heating, giving rise to an increase in molecular weight prior to cross-linked network structure. In order to prepare a sheet specimen of fully cross-linked phenolic resin, it is preferred to synthesize phenolic resin with a molecular weight higher than the oligomer and then to fabricate it into sheet suitable to friction experiment.

Thus, the above-obtained phenolic resin oligomer/ methanol mixture was heated with stirring at $120^{\circ} \mathrm{C}$ for about 10 min until an abrupt increase in melt viscosity due to molecular weight increase. In order to remove water formed by the reaction and methanol contained in the mixture from the reaction system, the reaction was carried out in an open stainless steel vessel placed on an electrical heating plate in a hood.

Soon after the abrupt viscosity increase, the reaction mixture was cooled down to room temperature, giving rise to formation of solid phenolic resin with a negligible amount of cross-linked structure. The brittle product thus obtained was ground into fine powders. By the usual heat pressing method with a $0.3 \mathrm{~mm}$ thick spacer and stainless steel plates with a size of $6 \mathrm{~cm} \times 6 \mathrm{~cm}$ covered with polytetrafluoroethylene films, a sheet fabrication was carried out in a process of pressing the fine powders under a pressure of $9.8 \mathrm{kN}$ at $90^{\circ} \mathrm{C}$ for $5 \mathrm{~min}$, heating to $105^{\circ} \mathrm{C}$, pressure release at the temperature for a few min and then pressing at $170^{\circ} \mathrm{C}$ for $30 \mathrm{~min}$. The heat-pressed sheet was cooled down to room temperature.
Preparation of Phenolic Resin-based Nanocomposite with Carbon Nanotubes

Carbon nanotubes are hardly dispersed in many organic solvents. In fact, they are not dispersed but coagulated in methanol. An examination revealed dispersion of multiwalled carbon nanotube kindly supplied by Carbon Nanotube Research Insitute Co., Japan in hexafluoroisopropanol (HFIP) better than in methanol. Preparation of phenolic resin-based nanocomposite was therefore made by the following two methods of addition of the carbon nanotube with and without HFIP to the phenolic resin oligomer/ methanol mixture. A $0.2 \mathrm{wt} \%$ of the carbon nanotube suspension in HFIP was used for the first method and the carbon nanotube was also added to the mixture without using HFIP for the second method.

A given amount of the carbon nanotube suspension in HFIP was mixed with a given amount of the phenolic resin oligomer/methanol mixture at room temperature in a stainless steel vessel to give the contents of carbon nanotube in the final, fully cross-linked phenolic resin-based nanocomposites to be 1, 3,5, 10 and $15 \mathrm{wt} \%$. The mixture was further mixed with stirring at $140^{\circ} \mathrm{C}$ until an abrupt melt viscosity increase due to in-situ polymerization of the oligomer, accompanying evaporation of solvents and water formed. After cooling to room temperature, the resultant brittle product was ground to fine powders. Heat-pressed sheets of the fully cross-linked phenolic resin-based nanocomposites were prepared by the same method as mentioned above.

A given amount of the carbon nanotube was also added, without HFIP, to a given amount of the phenolic resin oligomer/methanol mixture. Preparation of fully cross-linked phenolic resin-based nanocomposites with carbon nanotube content of $1,3,5,10,15$ and $20 \mathrm{wt} \%$ were also made by the same method as for the case of HFIP.

\section{Friction Experiment}

A sheet of the phenolic resin-based composites with carbon nanotubes, which was adhered to a steel disk, was slid against an SUJ2 steel ball with diameter of $4.76 \mathrm{~cm}(3 / 16 \mathrm{inch})$ by rotating the disk at a speed of $19.1 \mathrm{~cm} / \mathrm{sec}$ under a load of $1.96 \mathrm{~N}$ in a ball-on-disk friction machine at room temperature, recording friction coefficient as a ratio of frictional force to load.

\section{Transmission Electron Microscopy (TEM)}

A drop of suspension of carbon nanotubes in HFIP was placed onto a thin carbon film-coated $\mathrm{Cu}$ grid and the nanotubes without metal shadowing were subjected to TEM observation.

A TEM specimen of the phenolic resin-based nanocomposite was also prepared by the ultrathin 
cross-sectioning method using a diamond knife in a Reichert UltraCut S ultramicrotome.

In order to examine the worn surface morphology by TEM, a carbon replica film of the worn surface was prepared according to the method reported by Komoto et $a l .{ }^{9}$ A part of the wear track was carbonshadowed at an angle of $30^{\circ}$ by arc discharge of carbon electrode in a JEOL JEE-400 vacuum evaporator. The coated carbon surface was etched by low temperature plasma in air at a pressure of $c a$. 0.1 Torr (13.3 Pa). A drop of concentrated aqueous solution of gelatin was placed onto the plasma-treated surface. After complete drying, the hard gelatin, to which were adhered the coated carbon and the fine wear powders, was peeled from the worn surface and then placed upside down onto aqueous $10 \mathrm{wt} \% \mathrm{KSCN}$ solution to dissolve gelatin. As a result, a very thin carbon replica film, to which fine wear powders were adhered, was floated on the solution after dissolution of gelatin. The carbon replica film was then transferred onto water by floating it on aqueous film formed in a metal ring to wash gelatin away. After repeated washing, the carbon replica film was collected on a $\mathrm{Cu}$ grid and subjected to TEM observation.

TEM observation was carried out at an accelerating voltage of $80 \mathrm{kV}$ in a JEOL $1200 \mathrm{EX}$ transmission electron microscope.

\section{Scanning Electron Microscopy (SEM)}

Morphology of the wear track formed on the sheet specimen, which had been coated by $20 \mathrm{~nm}$ thick Pt, was examined at an accelerating voltage of $2.0 \mathrm{kV}$ in a JEOL JSM-6700F field-emission scanning electron microscope.

\section{Vickers Hardness Measurement}

Vickers hardness of the sheet specimen was measured by loading a $1.96 \mathrm{~N}$ for $20 \mathrm{~s}$ with a Vickes indentor onto the specimen with size of $30 \mathrm{~mm} \times 30 \mathrm{~mm}$ and thickness of $2 \mathrm{~mm}$ in an Akashi HM-112 Vickerstype of microhardness tester.

\section{RESULTS AND DISCUSSION}

\section{Morphology of Carbon Nanotubes and the Nanocom- posites}

In order to examine the morphology of the carbon nanotube used in this study, TEM observation was made. Figure 1 shows a TEM micrograph of the specimen obtained from a suspension in HFIP, indicating a mixture of rod-like nanotubes and ill-shaped granules. The nanotubes have rod thickness of $20-30 \mathrm{~nm}$ and wall thickness as large as $5-10 \mathrm{~nm}$. It is also seen that the nanotubes have end-capped structure. This result suggests end-capped multi-walled carbon nanotubes.

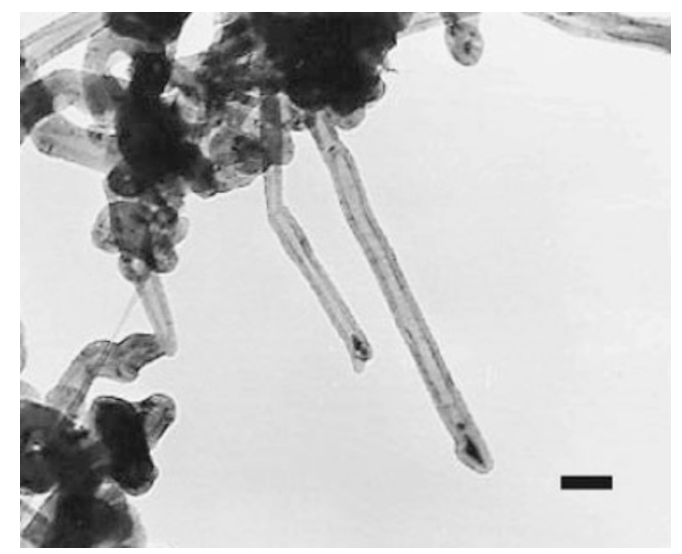

Figure 1. TEM micrograph of carbon nanotubes obtained from a suspension in HFIP.

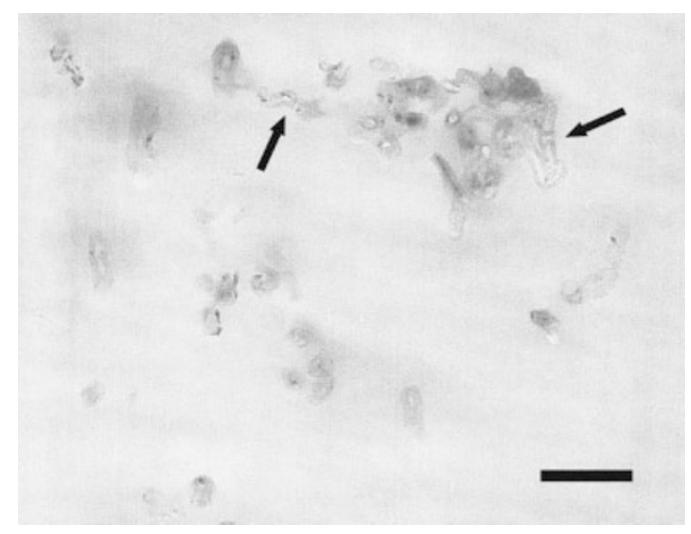

(a)

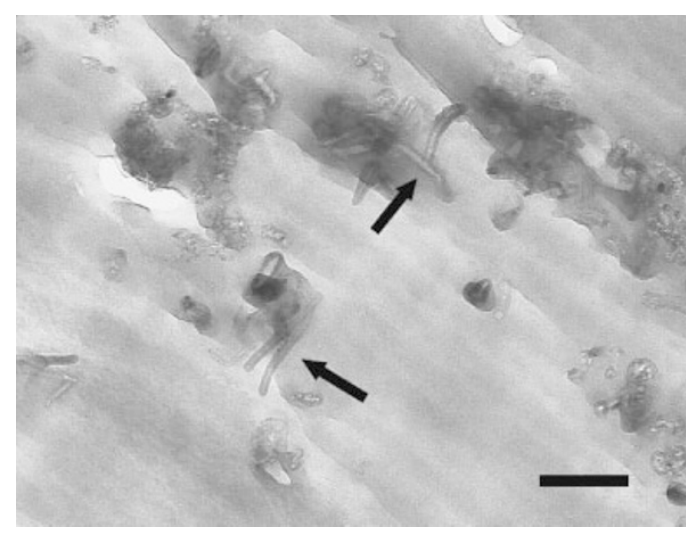

(b)

Figure 2. TEM micrographs of ultrathin sections of phenolic resin composites with carbon nanotube content of 1 (a) and 10 (b) wt $\%$. Scale bar: $200 \mathrm{~nm}$.

Morphology of the Phenolic Resin-based Nanocomposites with Carbon Nanotubes

Figure 2(a) shows TEM micrographs of ultrathin sections of the nanocomposites containing $1 \mathrm{wt} \%$ of carbon nanotubes which were prepared without HFIP. In the micrograph, carbon nanotubes are seen dark, as shown by arrows, due to absorption contrast, 


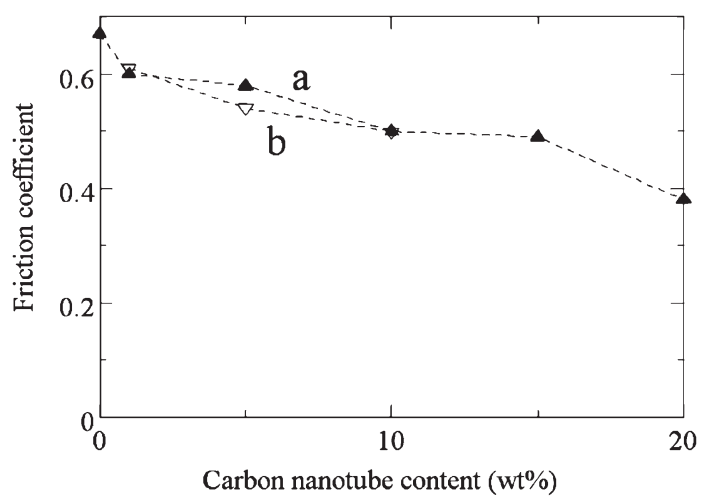

Figure 3. Friction coefficient of the phenolic resin nanocomposites as a function of carbon nanotube content, where the composites were prepared with (a) and without (b) HFIP.

indicating nearly homogeneous dispersion of the nanotubes in the phenolic resin matrix, irrespective of the dispersion medium. Figure 2(b) shows a TEM micrograph for a content of $10 \mathrm{wt} \%$ carbon nanotubes. This also indicates highly homogeneous dispersion of nanotubes in the matrix, except for the content.

The high dispersion of carbon nanotubes in the nanocomposites irrespective of the dispersion medium and the nanotube content is thought to be obtained by prevention of coagulation of the nanotubes due to viscosity increase of the matrix as the polymerization proceeds, accompanying evaporation of methanol and water formed by the reaction.

\section{Friction Behavior of the Nanocomposites}

Friction experiments were carried out for $4 \mathrm{~h}$, where nearly constant friction coefficients were obtained after $1 \mathrm{~h}$ for all the nanocomposites. The mean friction coefficient after $1 \mathrm{~h}$ is plotted against carbon nanotube content in Figure 3. Friction coefficient of the neat phenolic resin sheet without carbon nanotubes was as high as 0.67 . Friction coefficient decreased with increasing carbon nanotube content. It is noted that a load dependence of friction coefficient was almost same between the composites prepared with and without HFIP as the dispersion medium of carbon nanotubes. This result coincides with a similar morphology between the corresponding composites with and without HFIP as was mentioned above.

\section{SEM Morphology of Worn Surface}

SEM observations of the worn surfaces of the nanocomposites were made. The worn surface morphology of the nanocomposites was not dependent on the dispersion medium but on the carbon nanotube content, being consistent with the results obtained from TEM and friction experiment. In this paper, the worn surface morphology will be mentioned only for the nanocomposites prepared without using HFIP.
SEM micrographs of a low magnification, though not shown here, for the worn surfaces of the nanocomposites prepared without using HFIP indicated that an apparent worn surface roughness was largest for the neat resin and decreased with increasing carbon nanotube content.

In order to clarify the effect of carbon nanotube content on frictional behavior of the nanocomposites, morphology of their worn surfaces was examined by SEM at a high magnification. Figure 4(a) shows worn surface morphology of the neat phenolic resin, where an arrow indicates the sliding direction of the counter surface of a steel ball. In the micrograph are seen three typical textures: region A consisting of crevasses and discrete flat surface regions of $c a .1 \mu \mathrm{m}$ wide and a few micrometers long, region $\mathrm{B}$, a part of which is nearly removed from the matrix, accompanying crack formation, and region $\mathrm{C}$ with smooth surface which seems below the levels of regions A and B.

Compared with fibrous and other textures ${ }^{10-14}$ typical of thermoplastic polymers such as ultrahigh molecular weight polyethylene and others, the abovementioned particular textures should be formed by a friction and wear mechanism relevant to the crosslinked structure of phenolic resin. That is, no slippage of molecular chains takes place by friction for the cross-linked phenolic resin. This should give rise to chain scissions due to a large structural distortion within texture of several micrometers in size and then to initiation and propagation of crazes.

It can be said, therefore, that the crevasses and discrete flat surface regions were formed in the region A of Figure 4(a) by the mechanism mentioned above. A further sliding of the counter steel ball on the region $\mathrm{A}$ is thought to lead to wear of the discrete flat surface regions and formation of similar texture with more shallow crevasses. In region B, where a thin flat surface is easily destroyed by shear and indentation of the steel ball, giving rise to crack formation. Thus, the crack formation and craze propagation can cause formation of region $\mathrm{C}$ with a smooth surface. The surface of region $\mathrm{C}$ would be changed to a surface similar to that of region $\mathrm{A}$ in the subsequent stages of friction and wear.

Figure 4(b) shows a SEM micrograph of a worn surface of the composite containing $5 \mathrm{wt} \%$ carbon nanotube, indicating a surface roughness less than that (Figure 4(a)) of the neat phenolic resin. In Figure 4(b), are seen neither obvious crack textures nor smooth surface textures, but textures with somewhat round edges. Figure 4(c) shows a corresponding SEM micrograph for the composite containing $20 \mathrm{wt} \%$ of carbon nanotube, indicating a smooth worn surface. 


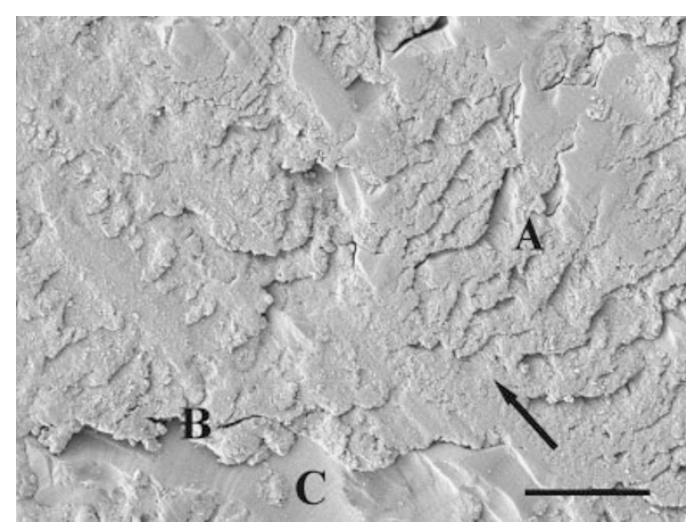

(a)

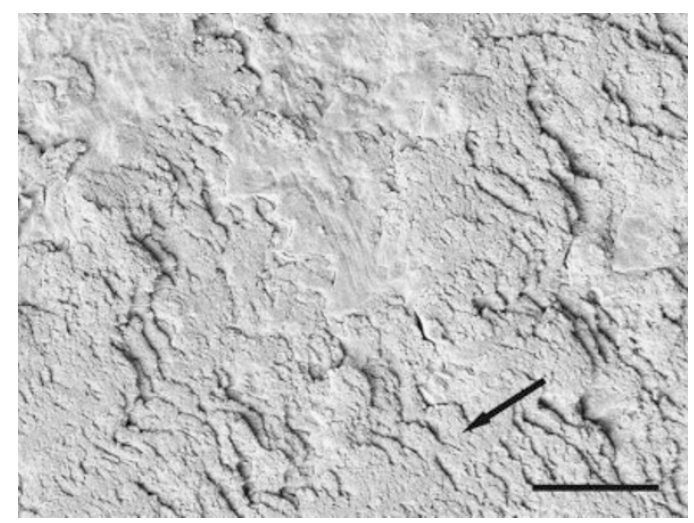

(b)

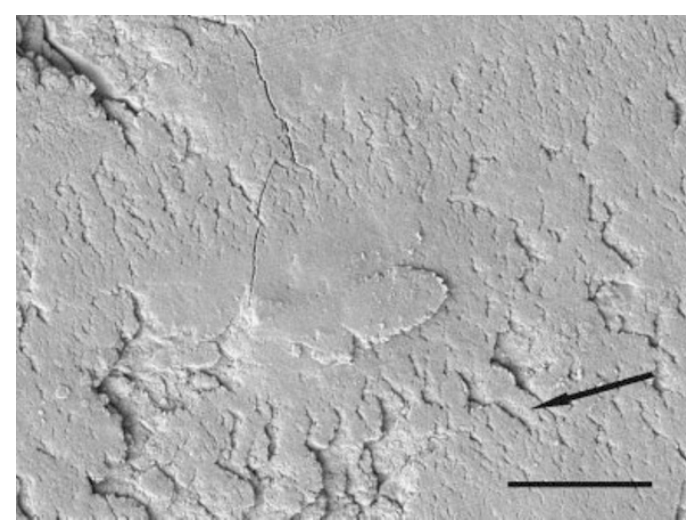

(c)

Figure 4. SEM micrographs of worn surfaces of the phenolic resin nanocomposites with carbon nanotube content of 0 (a), 5 (b) and 20 (c) wt \%. Arrows indicate the sliding direction of the steel ball. Scale bar: $5 \mu \mathrm{m}$.

\section{TEM Morphology of Worn Surface}

A high SEM contrast is principally obtained by edge effect, i.e. an enhanced amount of secondary ions generated from such rough surface edges as in Figure 4(a), while a low contrast is obtained from smooth surface because of less edge effect. As compared with little edge effect in SEM morphology of rather smooth worn surface, TEM morphology of carbon replica film, which is obtained by absorption contrast from

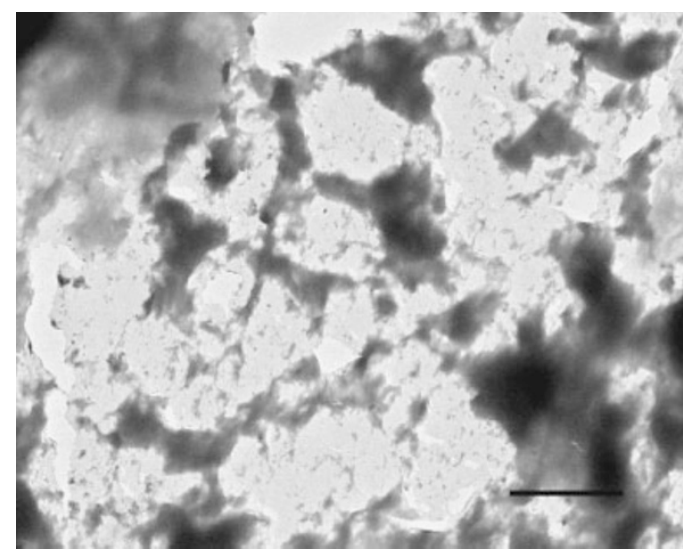

(a)

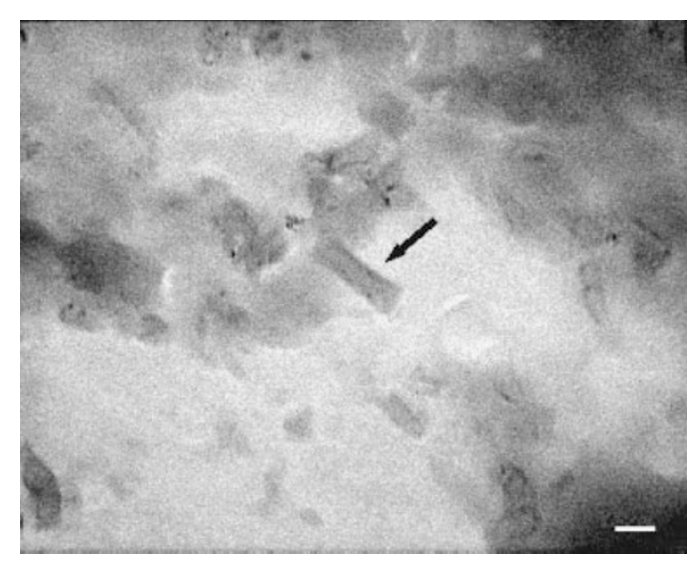

(b)

Figure 5. TEM micrographs of carbon replica films obtained from the worn surface of the phenolic resin nanocomposite with carbon nanotube content of $20 \mathrm{wt} \%$. Scale bar: $5 \mu \mathrm{m}$ (a) and $50 \mathrm{~nm}(\mathrm{~b})$.

fine wear powders adhered to the film, is preferred to examine the details of the smooth worn surface. Figure 5(a) shows a TEM micrograph of carbon replica film for worn surface of the phenolic resin composite with carbon nanotube content of $20 \mathrm{wt} \%$. This surface morphology almost corresponds to SEM morphology (Figure 4(c)) for the composite with carbon nanotube content of $20 \mathrm{wt} \%$. Figure 5(a) clearly indicates that ill-shaped fine wear powders seen dark are formed on the bright resin matrix. It can be said that such primary wear powders of less than $1 \mu \mathrm{m}$ are formed and then coagulated with each other in the course of sliding. This indicates that the primary wear powders decrease in size with increasing carbon nanotube content, giving rise to a change in worn surface morphology as a function of carbon nanotube content.

Figure 5(b) shows a TEM micrograph of a higher magnification for carbon replica film obtained from the worn surface of the composite with carbon nanotube content of $20 \mathrm{wt} \%$, where arrows indicate carbon nanotubes. This morphology indicates that long 


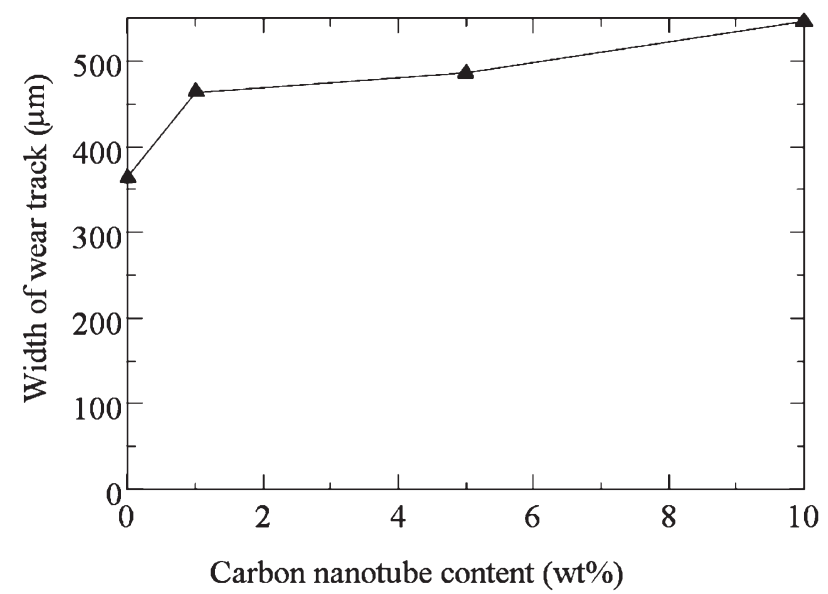

Figure 6. Width of wear track as a function of carbon nanotube content for the phenolic resin composites.

carbon nanotubes seen in Figure 1 were broken into short ones.

\section{Wear Behavior}

In order to examine the wear behavior of the nanocomposites, SEM observations were also made on the width of the wear track, as a measure of relative wear rate. Figure 6 shows a carbon nanotube content dependence of width of wear track formed after $4 \mathrm{~h}$ sliding of steel ball on the nanocomposite sheet. Supposed that a shape of the cross section of the wear track is arc throughout the wear process, the larger the wear track width, the higher the wear amount is. As is evident from Figure 6, a gradual increase in wear track width from $c a .360 \mu \mathrm{m}$ for the neat resin to $c a$. $550 \mu \mathrm{m}$ for the composite containing $10 \mathrm{wt} \%$ carbon nanotube suggests an increase in wear rate of the nanocomposite with increasing carbon nanotube content. It is noted that a large increase in wear track width was found between $360 \mu \mathrm{m}$ for the neat resin and $470 \mu \mathrm{m}$ for the composite with carbon nanotube content of $1 \mathrm{wt} \%$.

Surface hardness of the composites, which may be closely related to the wear behavior, was then estimated in terms of Vickers hardness. Vickers hardness was $686 \mathrm{MPa}$ for the neat phenol resin, while it was as low as $c a$. $390 \mathrm{MPa}$ for the composites with carbon nanotube content of 1 and $5 \mathrm{wt} \%$. This abrupt decrease in Vickers hardness by addition of a small amount of carbon nanotubes may coincides with a large increase in wear track width for these composites.

It may be said from these results that the deterioration of the phenol resin hardness by incorporation of carbon nanotubes gives rise to acceleration of wear of the resin. This may also suggest that carbon nanotubes located not only in the near surface but also in the deep surface play a role in formation of microcracks of the phenol resin therefrom.

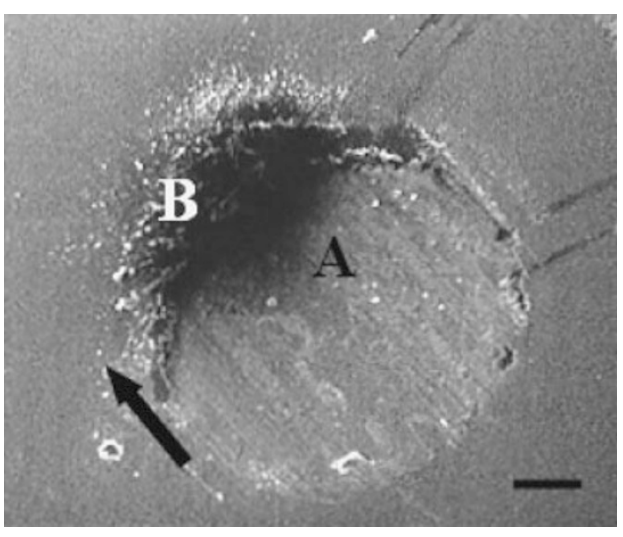

(a)

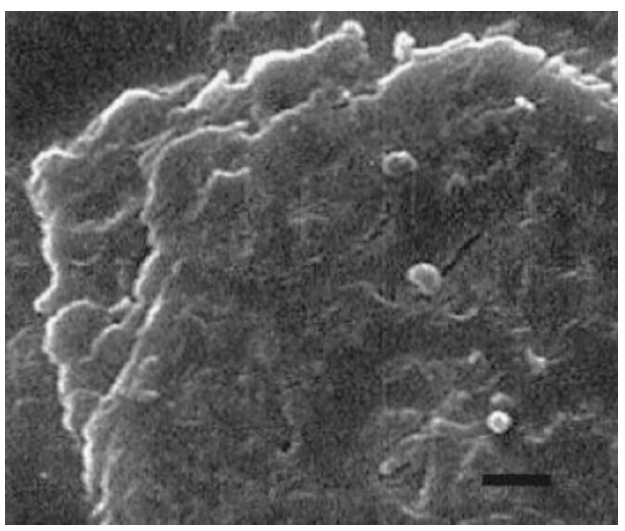

(b)

Figure 7. SEM micrographs of the counter steel ball surface obtained after $4 \mathrm{~h}$ sliding on the neat phenolic resin: whole contact region (a) and the central part (b). For A and B, see text. Arrow indicates the sliding direction of the resin surface. Scale bar: $100 \mu \mathrm{m}$ (a) and $5 \mu \mathrm{m}$ (b).

\section{Morphology of Steel Ball Surface}

In order to examine the detail in wear mechanism, SEM observation was also made on the counter surface, i.e., the surface of steel ball after the friction experiment. Figure 7 shows SEM micrographs of a steel ball surface after $4 \mathrm{~h}$ sliding on the neat phenol resin. Figure 7(a) shows a circular texture with a diameter of $c a .500 \mu \mathrm{m}$ formed on the ball, the diameter being larger than the wear tack width of $360 \mu \mathrm{m}$. This difference can be accounted for by deposition, i.e., transfer of phenolic resin wear powders onto the steel ball surface. In fact, ill-shaped wear powders shown in Figure 7(b) were found over the whole region of the circular texture. In other words, the wear powders are thought to be transferred onto a region larger than the load-applied contact area. A major amount of wear powders were deposited on the rear periphery of the circular texture. This result indicates that the friction and wear of the composites against steel ball mainly took place between the matrix composite and the transferred wear powders deposited on the steel ball. 


\section{A. IgARASHI et al.}

The size of transferred powder region on the steel ball decreased with increasing carbon nanotube content such that the size was $c a .400$ and $300 \mu \mathrm{m}$ for the composite with carbon nanotube content of 1 and $10 \mathrm{wt} \%$, respectively, though their SEM micrographs are not shown. This is contrary to a tendency of the increase in wear track width with increasing carbon nanotube content. It can be said, therefore, that the less the carbon nanotube content is, the less the amount of transferred wear powders.

\section{REFERENCES}

1. T. Komoto, in "Automobiles and Textiles in Future," K. Iizuka, A. Hinata, T. Matsuo, O. Yaida, Y. Yamazaki, and A. Yonenaga, Ed., Sen-isha, Osaka, 2004, chapt. 6, p 190.

2. K. Haraguchi, Y. Usami, and Y. Ohno, J. Mater. Sci., 33,
3337 (1998).

3. K. Haraguchi, Y. Usami, K. Yamamura, and S. Matsumoto, Polymer, 39, 6243 (1998).

4. K. Haraguchi, Tribologist, 45, 36 (2000).

5. T. Nisino, Kobunshi, 51, 32 (2002).

6. P. J. F. Harris, Int. Mater. Rev., 49, 31 (2004).

7. M. Takenaka, Polymer Digest, 55, 52 (2003).

8. N. Ohtake, K. Enomoto, and T. Yasuhara, New Diamond, 19, 12 (2003).

9. T. Komoto, Y. Kumashiro, Y. Nakamura, Y. Watanabe, and T. Ohsima, Kobunshi Ronbunshu, 49, 383 (1992).

10. T. Komoto and S. Hironaka, Angew. Makromol. Chem., 150, 189 (1987).

11. T. Komoto, Seikei Kako, 9, 948 (1997).

12. S. Hironaka, T. Komoto, and K. Tanaka, Wear, 87, 85 (1983).

13. S. Takamatsu, T. Kobayashi, T. Komoto, M. Sugiura, and K. Ohara, Sen-i Gakkaishi, 50, 550 (1994).

14. T. Komoto, Tribologist, 45, 19 (2000). 